\title{
Ferromagnetism of an all-carbon composite composed of a carbon nanowire inside a single-walled carbon nanotube
}

\author{
Xiaoping Yang \\ National Laboratory of Solid State Microstructures and Department of Physics, \\ Nanjing University, Nanjing 210093, China, \\ and Department of Physics, Huainan Normal University, Huainan, Anhui 232001, China \\ Jinming Dong \\ National Laboratory of Solid State Microstructures and Department of Physics, \\ Nanjing University, Nanjing 210093, China
}

(Dated: 16 July 2007)

\begin{abstract}
Using the first-principles spin density functional approach, we have studied magnetism of a new type of all-carbon nanomaterials, i.e., the carbon nanowires inserted into the single-walled carbon nanotubes. It is found that if the 1D carbon nanowire density is not too higher, the ferromagnetic ground state will be more stable than the antiferromagnetic one, which is caused by weak coupling between the $1 \mathrm{D}$ carbon nanowire and the single-walled carbon nanotube. Also, both dimerization of the carbon nanowire and carbon vacancy on the tube-wall are found to enhance the magnetic moment of the composite.
\end{abstract}

PACS numbers: 73.22.-f, 75.75.+a, 61.46.+w, 71.15.Nc 
All allotropes of carbon have long been considered as the most promising candidates of the magnets [1]. Recent discovery of weak ferromagnetism in pure carbon systems, such as carbon foam, graphite, oxidized $\mathrm{C}_{60}$ and polymerized rhombohedral $\mathrm{C}_{60}$ [2-5], has stimulated renewed interests in new possible magnetic materials made of only carbon. And also a lot of theoretical works on the magnetism in all-carbon materials have been done [6-8].

Even so, the origin of ferromagnetism has not yet been clear. The most important question is whether the spin polarization is induced intrinsically or by magnetic impurities. If intrinsic, what causes the unpaired spins, and how do they couple together ferromagnetically? There are several theoretical models for the magnetism in carbon-based compounds, which are nonbonding localized states at the zigzag edge [6,9], carbon vacancies or defects $[4,7,10]$, and the trivalent carbon radicals [8].

The carbon nanotubes (CNTs) have attracted much attention since their discovery [11] due to their remarkable electronic and mechanical properties, and seem promising for future nanoscale electronic devices [12]. A little change of the CNT structure, e.g., the topological defect on tube wall, can greatly alter its electronic properties, and the same holds when impurity atoms are encapsulated into the CNT. Recently, X. Zhao et al. discovered a new type of 1D carbon structures, carbon nanowires (CNWs), formed by a linear carbon chain inserted into the innermost tube $(\sim 7.0 \AA$ diameter $)$ of a multiwalled CNT [13]. On the other hand, however, the CNT magnetic properties have been less studied [14], and so far, no experimental observations show magnetism in CNTs.

In this letter, we investigate the electronic and magnetic properties of the all-carbon composite, CNW@SWNT shown in Fig. 1(a), which is assumed to be composed of a CNW inserted into the core of a single-walled CNT (SWNT). Our most striking result is to find the flat-band ferromagnetism in various CNW@SWNTs at the lower enough CNW densities, which is different from the previous theoretical mechanisms [4,6,7-10].

Our theoretical calculations were performed with the total energy plane-wave pseudopotential program CASTEP [15] in the local spin density approximation (LSDA), in which the Ceperley-Alder form exchange-correlation energy [16] was included. The ion-electron interaction for the carbon atoms is modeled by the Vanderbilt ultra-soft local pseudopotentials [17] with plane wave cut-off energy of $240 \mathrm{eV}$. The CNW@SWNT is treated in a supercell geometry [18] with intertube distance of $16 \AA$, being larger enough to prevent tube-tube interactions, and the supercell is optimized by employing BFGS geometry optimization scheme [19].

We choose the armchair $(5,5)$ tube as the outer tube because its diameter is the closest to that of the innermost tube observed in the experiment [13]. The calculated band structures of the pure $(5,5)$ tube, and the CNW@ $(5,5)$ (model I in Fig. 1) are shown in Fig. 2(a) and 2 (b), respectively. The most interesting is appearance of two flat-bands around $E_{F}$ with the first one doubly degenerate, and their widths of about 0.05 and $0.4 \mathrm{eV}$, respectively, which are clearly seen from Fig. 2(b), and are absent in Fig. 2(a), demonstrating they are produced mainly by the CNW. Since the CNW period of $4.89 \AA$ is larger than the distance of $3.4 \AA$ between the graphite layers, the direct coupling between the CNW atoms can be neglected. Thus, the narrow flat-bands can be induced only by the weak coupling between the CNW and the tube. As shown below, the flat-bands will cause the higher density of states (DOS) at the $E_{F}$, and if the interaction between the band electrons is introduced, the FM ordering would be induced, which was proved exactly in the Hubbard model under certain conditions [20].

The total DOS and partial DOS (PDOS) of the CNW@ $(5,5)$ are shown in Fig. 2c, 
d, e. It is seen from Fig. 2(c) that there exist higher DOSs at and just below the $E_{F}$, which are almost totally contributed by the CNW, identified by comparing Fig. 2(d) with $2(\mathrm{e})$. More importantly, they are completely spin polarized, making the model I composite a ferromagnetic (FM) metal with a total magnetic moment of $2.44 \mu_{B}$ in its one unit cell, which is mainly contributed by the CNW atoms. In order to get its real ground state, we then made the same LSDA calculation on a doubled unit cell, and found the antiferromagnetic (AFM) state is higher in energy than the FM one by $73.8 \mathrm{meV}$ per Model I unit cell, meaning the FM state is its ground state.

We also made the same LSDA calculations on other CNW@(5,5) with different CNW periods, e.g., $1 / 2,1$ and $4 / 3$ times that of the pure $(5,5)$ tube, respectively, and found that only if the CNW density is not too higher, can the weak coupling between the CNW and (5, 5 ) tube induce the flat-bands at the $E_{F}$, producing thus magnetic moments. For example, in the case of $1 / 2$, the flat-band will disappear, and so no ferromagnetism in its corresponding composite of $\mathrm{CNW} @(5,5)$. But, all of others have the flat-bands and the magnetic moments.

It is well known that the Coulomb correlations will affect the electronic states of weakly bonded carbon atom (here, e.g., the CNW atoms), hence the magnetic property of the system. Therefore, we have also made the LSDA+U calculation on the model I CNW@ $(5,5)$ by using the projector augmented wave (PAW) potentials in the VASP code [21,22]. In our calculations, the on-site Coulomb repulsion $\mathrm{U}=3.0 \mathrm{eV}$, and the exchange $\mathrm{J}=0.9 \mathrm{eV}$ have been taken only for the CNW atoms. It is found that the FM state with a magnetic moment of $2.42 \mu_{B}$ per Model I unit cell is lower in energy than the AFM one by $53.5 \mathrm{meV}$ per Model I unit cell, meaning the FM state is always the ground state of the system.

The ferromagnetism found in the CNW@SWNTs reveals realization of the flat-band ferromagnetism in the all-carbon composites with only $s$ and $p$ orbitals. Analyses of the orbital density distributions in the flat-band states show that they are composed mainly of the $p$ orbitals of CNW atoms and less of the $\pi$ orbitals of tube-wall atoms. And they are localized around the CNW atoms, but at the same time, extended along the tube direction through the weak coupling between the CNW atoms and the SWNT, which is clearly demonstrated by the small dispersion of the flat-band shown in Fig. 2(b). Therefore, if the spins are parallel, the repulsive interaction energy between the flat-band electrons would be reduced on average, compared with the case of anti-parallel spins, because of the Pauli exclusion, favoring obviously more FM state than AFM one, which has also been proved by the LSDA+U calculation mentioned above. In order to clarify more certainly the mechanism for the ferromagnetism due to weak coupling between the tube and the wire, a same LSDA+U calculation on the isolated CNW in Model I (in Fig. 1(a)) has been made, demonstrating the FM state is higher in energy than the AFM one by $111 \mathrm{meV}$ per CNW atom, meaning the AFM state is the ground state of the isolated CNW, in which the on-site Coulomb repulsion of $\mathrm{U}=3.0 \mathrm{eV}$, and the exchange $\mathrm{J}=0.9 \mathrm{eV}$ have been used.

What is effect of varous outer SWNTs with different diameters or chiral symmetries on the magnetism of the composites? We select two different CNW@SWNTs, such as CNW@(9,0) and CNW@(8,0), with fixed CNW periods, which are the same as those of $(9,0)$ and $(8$, $0)$ tubes, respectively. By the same LSDA calculations we found that both of them have magnetism with the magnetic moment of $2.41 \mu_{B}$ and $2.24 \mu_{B}$ per composite unit cell, respectively, demonstrating no significant effects from a little bit larger or smaller SWNT diameter and different chiral angles of the outer tubes. So, the CNW@SWNTs composites may find wide-scale technological applications.

Due to the well known Peierls instability, we found by the first-principles LDA calculation 
that the dimerized phase of the model I is lower in energy than the undimerized one by 81.6 meV per model I unit cell, whose final optimization structure is shown in Fig. 1 too, called as model II. In addition, we study also the possible effect of the defect in real materials by introducing carbon vacancy on the tube wall in model II, and its optimized structure is called as model III in Fig. 1. For comparison, we list in Table I all related LSDA results for the three models in Fig. 1, from which, it is obvious that the FM behavior appears in all the three composites. The model III has the largest magnetic moment, which not only mainly comes from CNW atoms, but also is partly contributed by the carbon atoms on the tube wall around the vacancy. In addition, the charges in model III are transferred not only from tube to the dimerized CNW, but also from some atoms to the others on the same tube wall. The transferred charges between the tube-wall atoms are mostly localized around the vacancy atom, leading to appearance of the magnetic moment of $-0.04 \sim 0.9 \mu_{B}$ per carbon atom on the tube wall, which is absent at all in model I or II.

Finally, we show the spin densities $\left[n_{\uparrow}(\mathbf{r})-n_{\downarrow}(\mathbf{r})\right]$ (the up spin is the majority) of three models in Fig. 3, from which it is clearly seen that in model I, the spin density higher than 0.0068 electrons $/ \AA^{3}$ exists only at the $\mathrm{CNW}$ atoms, and in model II, it extends into the space between two nearer CNW atoms. In model III, however, the same magnitude spin density can also be found at the tube atoms nearby the vacancy. So, both the CNW dimerization and the vacancy defect on the tube wall can enhance the ferromagnetism of the composite.

In summary, we have studied several all-carbon CNW@SWNTs by the first-principles LSDA and LSDA $+U$ calculations, and identified realization of the flat-band ferromagnetism in the composites if the 1D CNW period is larger enough, which is caused by the weak coupling between the 1D CNW and the outer tube. In addition, it is found that the CNW dimerization and carbon vacancy on the tube wall enhance the ferromagnetism of the composite. Our results indicate that the $\mathrm{CNW}$ inside carbon nanotube is a promising new candidate for the all-carbon nanometer-ferromagnets.

This work was supported by the Natural Science Foundation of China under Grant No. A040108 and No. 90103038. X. P. Yang thanks Mr. J. Zhou and H. M. Weng for their help in the numerical calculations. 
[1] T.L. Makarova, cond-mat/0207368.

[2] A.V. Rode, E.G. Gamaly, A.G. Christy, J.G. Fitz Gerald, S.T. Hyde, R.G. Elliman, B. LutherDavies, A.I. Veinger, J. Androulakis, and J. Giapintzakis, Phys. Rev. B 70, 054407 (2004).

[3] P. Esquinazi, A. Setzer, R. Hőhne, C. Semmelhack, Y. Kopelevich, D. Spemann, T. Butz, B. Kohlstrunk, M. Lősche, Phys. Rev. B 66, 024429 (2002), Phys. Rev. Lett. 91, 227201 (2003); J.M.D. Coey, M. Venkatesan, C.B. Fitzgerald, A.P. Douvalis, I.S. Sanders, Nature (London) 420, 156 (2002).

[4] Y. Murakami and H. Suematsu, Pure Appl. Chem. 68, 1463 (1996).

[5] T.L. Makarova, B. Sundqvist, R. Hőhne, P. Esquinazi, Y. Kopelevich, P. Scharff, V.A. Davydov, L.S. Kashevarova, A.V. Rakhmanina, Nature (London) 413, 716 (2001); R.A. Wood, M H Lewis, M R Lees, S M Bennington, M G Cain, N Kitamura, J. Phys. CM 14, L385 (2002).

[6] K. Kusakabe and M. Maruyama, Phys. Rev. B 67, 092406 (2003).

[7] A.N. Andriotis, M. Menon, R.M. Sheetz, L. Chernozatonskii, Phys. Rev. Lett. 90, 026801 (2003).

[8] N. Park, M. Yoon, S. Berber, J. Ihm, E. Osawa, D. Tománek, Phys. Rev. Lett. 91, 237204 (2003).

[9] M. Fujita, K. Wakabayashi, K. Nakada, K. Kusakabe, J. Phys. Soc. Jpn. 65, 1920 (1996); K. Nakada, M. Fujita, G. Dresselhaus, M. Dresselhaus, Phys. Rev. B 54, 17954 (1996).

[10] P.O. Lehtinen, A.S. Foster, A. Ayuela, A. Krasheninnikov, K. Nordlund, R.M. Nieminen, Phys. Rev. Lett. 91, 017202 (2003).

[11] S. Iijima, Nature (London) 354, 56 (1991).

[12] R. Saito, G. Dresselhaus, M.S. Dresselhaus, Physical Properties of Carbon Nanotubes, Imperial College Press, London, 1998.

[13] X. Zhao, Y. Ando, Yi Liu, M. Jinno, T. Suzuki, Phys. Rev. Lett. 90, 187401 (2003).

[14] A. Oshiyama, S. Okada, S. Saito, Physica B 323, 21 (2002); Jin Choi, Y. Kim, K.J. Chang, D. Tománek, Phys. Rev. B 67, 125421 (2003).

[15] M.D. Segall, P.J.D. Lindan, M.J. Probert, C.J. Pickard, P.J. Hasnip, S.J. Clark, M.C. Payne, J. Phys.: Cond. Matt. 14, 2717 (2002).

[16] D.M. Ceperley and B.J. Alder, Phys. Rev. Lett. 45, 566 (1980).

[17] A.M. Rappe, K.M. Rabe, E. Kaxiras, J.D. Joannopoulos, Phys. Rev. B 41, 1227 (1990).

[18] X. Blase, L.X. Benedict, E.L. Shirley, S.G. Louie, Phys. Rev. Lett. 72, 1878 (1994).

[19] T.H. Fischer and J. Almlof, J. Phys. Chem. 96, 9768 (1992).

[20] E.H. Lieb, Phys. Rev. Lett. 62, 1201 (1989); 62, 1927 (1989); A. Mielke, J. Phys. A 24, L73 (1991); 24, 3311 (1991); 25, 4335 (1992); H. Tasaki, Phys. Rev. Lett. 69, 1608 (1992); Prog. Theor. Phys. 99, 489 (1998).

[21] G. Kresse, J. Hafner, Phys. Rev. B 47, 558 (1993); ibid 49, 14251 (1994); G. Kresse, J. Furhmuller, Software VASP, Vienna (1999); Phys. Rev. B 54, 11169 (1996); Comput. Mat. Science 6, 15 (1996).

[22] At the present time, the CASTEP code has not included the LSDA+U program, and so we used the VASP package to do the LSDA+U calculations. Generally the PAW potentials are more accurate than the ultra-soft pseudopotentials. 


\section{TABLE}

Table I. The LSDA-calculated magnetic moment of the FM state in three models of the CNW@ $(5,5)$ shown in Fig. 1.

\begin{tabular}{l|l|l|l}
\hline Model & $\begin{array}{l}\text { magnetic moment }\left(\mu_{B}\right) \text { per unit cell } \\
\text { of each Model }\end{array}$ & $\begin{array}{l}\text { magnetic moment }\left(\mu_{B}\right) \text { per } C N W \\
\text { atom }\end{array}$ \\
\hline I & 2.44 & 2.42 \\
\hline II & 5.04 & 2.40 \\
\hline III & 5.93 & 2.38 \\
\hline
\end{tabular}




\section{Figure Captions}

Fig. 1 (Color online). Ball-and-stick model of the different kinds of CNW@(5, 5). (a) The model I. Its CNW period is two times larger than that of the pure $(5,5)$ tube.

(b) The model II, showing a dimerized CNW. (c) The model III, which is the same as (b) except for existence of a carbon vacancy on tube-wall. The boxes represent the unit cells.

Fig. 2 (Color online). Calculated band structures for: (a) pure (5, 5) tube, (b) model I CNW@(5,5). And the DOS of the model I CNW@(5, 5): (c) Total DOS, (d) PDOS of the tube, and (e) PDOS of the CNW. The blue and red represent the majority (up) and minority (down) spin, respectively.

Fig. 3 (Color online). Spin density $\left[n_{\uparrow}(\mathbf{r})-n_{\downarrow}(\mathbf{r})\right]$ distributions in the composites shown in Fig. 1. (a) model I. (b) model II. (c) model III. The cyan clouds represent spin-densities exceeding 0.0068 electrons $/ \AA^{3}$. 


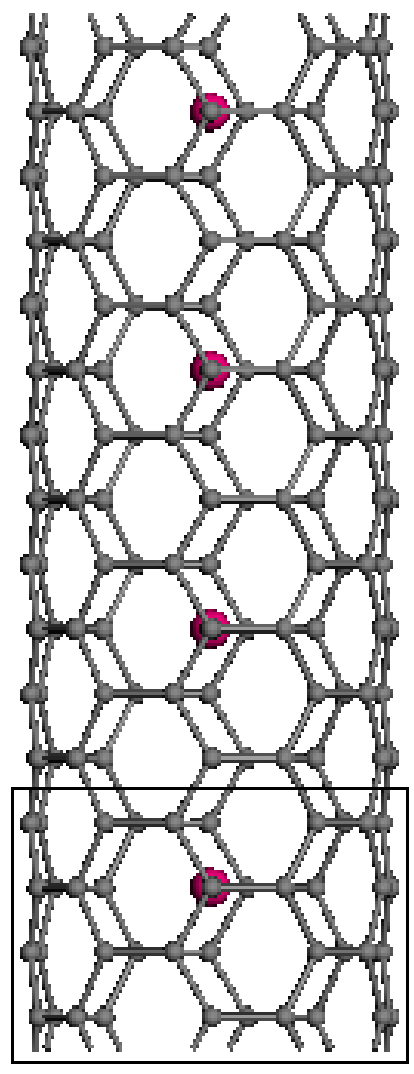

(a)

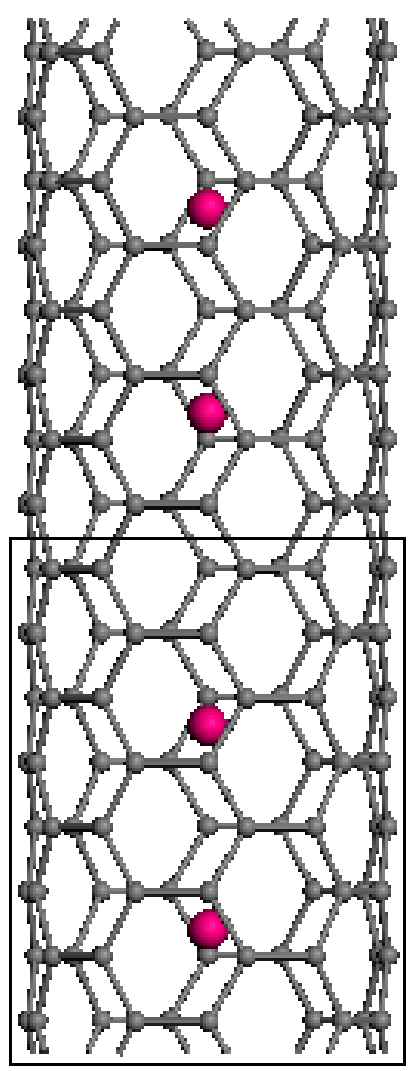

(b)

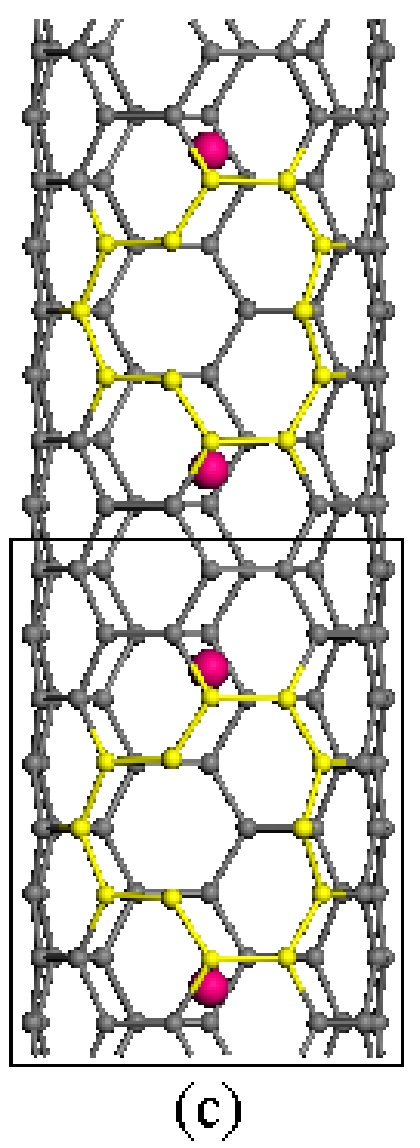

(c) 

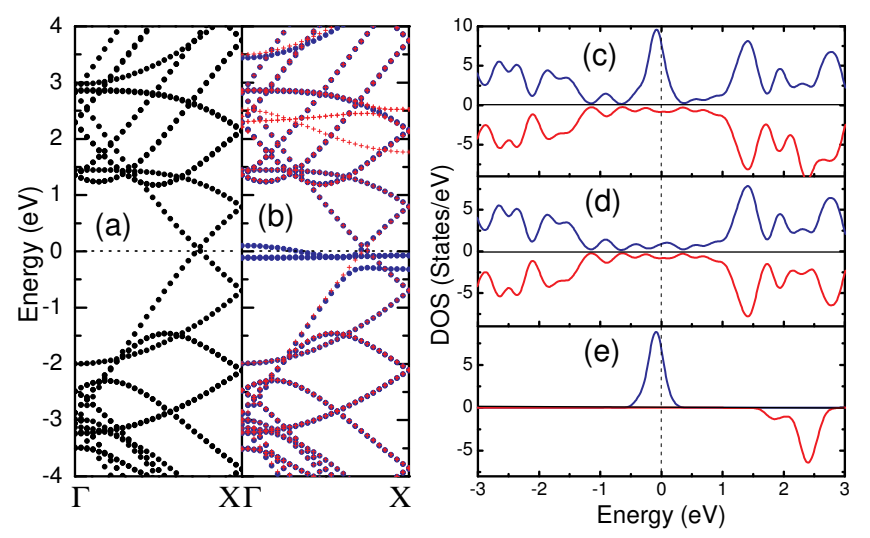


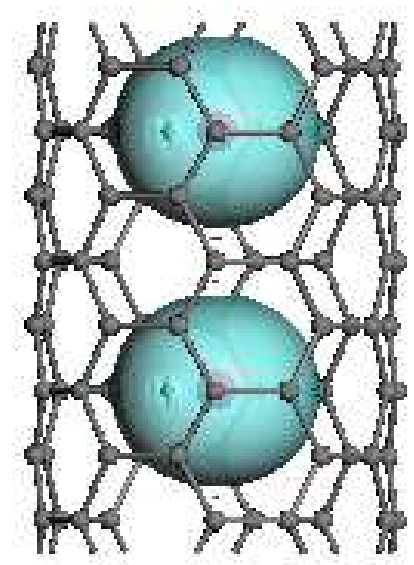

(a)

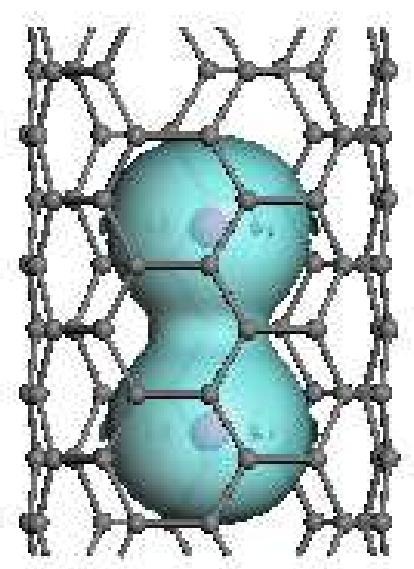

(b)

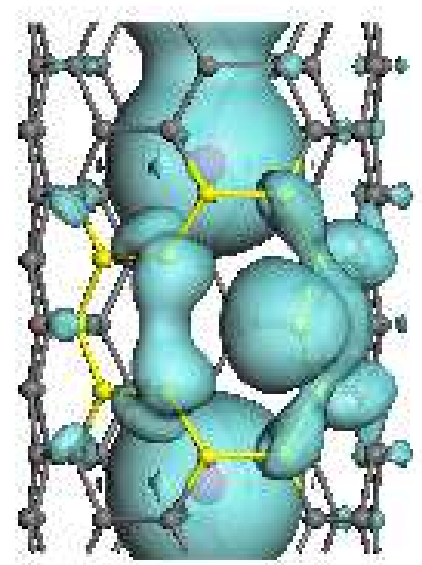

(c) 